\title{
Orosomucoid 1 Attenuates Doxorubicin-Induced Oxidative Stress and Apoptosis in Cardiomyocytes via Nrf2 Signaling
}

\author{
Xiaoli Cheng, ${ }^{1}$ Dan Liu, ${ }^{2}$ Ruinan Xing, ${ }^{2}$ Haixu Song, ${ }^{2}$ Xiaoxiang Tian, ${ }^{2}$ Chenghui Yan, ${ }^{2}$ \\ and Yaling Han $\mathbb{1 0}^{1}$ \\ ${ }^{1}$ Department of Cardiology, Shengjing Hospital of China Medical University, Shenyang, Liaoning Province 110004, China \\ ${ }^{2}$ Department of Cardiology and Cardiovascular Research Institute of PLA, General Hospital of Northern Theater Command, \\ Shenyang, Liaoning Province 110016, China
}

Correspondence should be addressed to Yaling Han; yaling.han1953@gmail.com

Received 16 June 2020; Revised 22 August 2020; Accepted 8 October 2020; Published 19 October 2020

Academic Editor: Roland E. Akhigbe

Copyright (c) 2020 Xiaoli Cheng et al. This is an open access article distributed under the Creative Commons Attribution License, which permits unrestricted use, distribution, and reproduction in any medium, provided the original work is properly cited.

\begin{abstract}
Doxorubicin (DOX) is an effective anticancer drug, but its therapeutic use is limited by its cardiotoxicity. The principal mechanisms of DOX-induced cardiotoxicity are oxidative stress and apoptosis in cardiomyocytes. Orosomucoid 1 (ORM1), an acute-phase protein, plays important roles in inflammation and ischemic stroke; however, the roles and mechanisms of ORM1 in DOXinduced cardiotoxicity remain unknown. Therefore, in the present study, we aimed to investigate the function of ORM1 in cardiomyocytes experiencing DOX-induced oxidative stress and apoptosis. A DOX-induced cardiotoxicity animal model was established in C57BL/6 mice by administering an intraperitoneal injection of DOX $(20 \mathrm{mg} / \mathrm{kg})$, and the control group was intraperitoneally injected with the same volume of sterilized saline. The effects were assessed after $7 \mathrm{~d}$. Additionally, H9c2 cells were stimulated with DOX $(10 \mu \mathrm{M})$ for $24 \mathrm{~h}$. The results showed decreased ORM1 and increased oxidative stress and apoptosis after DOX stimulation in vivo and in vitro. ORM1 overexpression significantly reduced DOX-induced oxidative stress and apoptosis in H9c2 cells. ORM1 significantly increased the expression of nuclear factor-like 2 (Nrf2) and its downstream protein heme oxygenase 1 (HO-1) and reduced the expression of the lipid peroxidation end product 4-hydroxynonenal (4-HNE) and the level of cleaved caspase-3. In addition, Nrf2 silencing reversed the effects of ORM1 on DOX-induced oxidative stress and apoptosis in cardiomyocytes. In conclusion, ORM1 inhibited DOX-induced oxidative stress and apoptosis in cardiomyocytes by regulating the Nrf2/HO-1 pathway, which might provide a new treatment strategy for DOX-induced cardiotoxicity.
\end{abstract}

\section{Introduction}

Heart failure is a prevalent disease worldwide, representing a severe manifestation and end stage of most heart diseases. The number of patients with heart failure in China has reached $1-2 \%$ of the total population. Heart failure not only significantly decreases the quality of life of patients and causes a substantial economic burden on patient families and society but is also associated with a high mortality rate. Over $50 \%$ of patients with chronic heart failure die within five years of diagnosis. Therefore, heart failure is also known as "the final battlefield of cardiovascular disease of the 21st century".

The incidence rate of cancer is more than one-third worldwide, and cardiovascular disease is the two causes of death in developed countries. Doxorubicin (DOX), a cardiotoxic anthracycline chemotherapy drug, was first isolated from Streptomyces peucetius caesius in 1967 [1,2]. DOX plays an important role in the treatment of many cancers, as $32 \%$ of patients with breast cancer, $57-70 \%$ of elderly patients with lymphoma, and $50-60 \%$ of children with cancer have been treated with anthracycline drugs [3-6]. DOX has been included in the World Health Organization (WHO) model list of essential medicines, as a milestone of cancer therapy development and one of the most commonly used antitumor anthracycline antibiotics.

However, the cardiotoxic effects of DOX limit its use in cancer patients with irreversible degenerative cardiomyopathy and heart failure. More than half of the elderly patients and children who survive lymphoma and other cancers, 
respectively, show a high risk of cardiotoxicity after DOX treatment [7]. Therefore, it is essential to study DOXinduced myocardial injury to increase survival rates and improve the quality of life of patients. Numerous studies have shown that oxidative stress and apoptosis are important mechanisms of DOX-induced myocardial injury [8, 9]. Therefore, an effective antioxidant and antiapoptotic agent that improves DOX-induced myocardial injury is urgently needed.

Orosomucoid 1 (ORM1) is an acute-phase protein that was first discovered by Tokita and Schmid more than 100 years ago. It is mainly synthesized in the liver, but many extrahepatic tissues have also been reported to produce ORM1 under various conditions [10, 11]. ORM1 performs various activities, acting as an acute-phase reactant and disease marker, regulating immunity, maintaining the capillary barrier function, regulating sphingomyelin metabolism, and scavenging reactive oxygen species (ROS) [12-14]. However, the current understanding of ORM1 is limited $[15,16]$, and its role in cardiovascular disease is not clear.

Nuclear factor-like 2 (Nrf2) is an important member of the cap collar family of basic leucine zipper transcription factors; it plays a role in the antioxidant defense system by regulating the expression of antioxidant enzymes [17, 18]. During conditions of oxidative stress, Nrf2 is activated, translocated to the nuclear region, and combined with the antioxidant response element located in the promoter region of phase II antioxidant enzyme genes, such as heme oxygenase 1 (HO- 1$)$. It results in the detoxification of 4 hydroxylnonenal (4-HNE) and a decrease in ROS level, which releases the level of apoptosis [19, 20]. In previous studies, it was found that DOX decreases the expression of Nrf2, resulting in an increase in oxidative stress and cell apoptosis [21]. However, it is not clear whether ORM1 plays a protective role during oxidative stress and cell apoptosis by regulating Nrf2.

In our study, we aimed to investigate the potential relationship between ORM1 and Nrf2 and clarify the roles of ORM1 in the oxidative stress and cell apoptosis resulting from DOX-induced cardiomyocyte toxicity.

\section{Materials and Methods}

2.1. Animals and In Vivo Experimental Design. Eight-weekold male C57BL/6J mice were acquired from Southern Animal Model Co., Ltd. (Nanjing, China) and housed in a nonpathogenic animal facility at an ambient temperature of $23 \pm 2^{\circ} \mathrm{C}$ and on a $12 \mathrm{~h}$ dark/light cycle for $2 \mathrm{wk}$. DOX (Sigma, USA) was dissolved in sterilized saline to a final concentration of $<2 \%$. Forty mice were randomly divided into the following two groups with 20 mice in each group: control (saline) and DOX (20 mg/kg DOX). Mice in the DOX group were intraperitoneally injected with $10 \mathrm{mg} / \mathrm{kg}$ DOX on the first and fourth days, leading to a cumulative DOX dose of $20 \mathrm{mg} / \mathrm{kg}$. Mice in the control group were intraperitoneally injected with the same volume of saline at the same times. These doses of DOX and sterilized saline were selected based on previous studies [22]. Seven days after the first DOX injection, all mice were euthanized. All animal experiments complied with the "Guiding Principles for the Care of Experimental Animals" and "Guidelines for the Care and Use of Experimental Animals" (NIH publication 86-23, revised 1985). Animal care and procedures were approved by the Committee on the Care and Use of Laboratory Animals of the General Hospital of Northern Theater Command.

2.2. Cell Culture, Transfection, and In Vitro Experimental Design. The H9c2 cell line was purchased from the Chinese Academy of Sciences, Shanghai Institute for the Cell Resource Center and cultured in $5 \% \mathrm{CO}_{2}$ at $37^{\circ} \mathrm{C}$. Adenoviral vectors (Ad-control and Ad-ORM1) were purchased from Hanbio Biotechnology (China). Nrf2 siRNA and control siRNA were purchased from Thermo Scientific (USA). H9c2 cells were seeded onto 6 -well plates $\left(2 \times 10^{5}\right.$ cells per well), and fetal bovine serum (FBS, USA) was added to $2 \mathrm{~mL}$ of normal culture medium without antibiotics. After the cells reached a confluence of $60-70 \%$, they were transfected using Lipo iMAX (Thermo Scientific, USA) to control siRNA or target siRNA double strands (100 pM). After $48 \mathrm{~h}$ of transfection, the cells were collected for further experiments. DOX was dissolved in sterile saline.

Before the experiment, sterile saline was used as a control. In the preliminary in vitro experiment, the cells were divided into the following four groups: (1) control, (2) ORM1 (100 MOI) treated (ORM1), (3) DOX $(10 \mu \mathrm{M})$ treated (DOX), and (4) DOX+ORM1 treated (DOX+ORM1). In the subsequent in vitro experiments, the cells were divided into the following groups: (1) control, (2) Nrf2 siRNA treated (Nrf2), (3) DOX treated (DOX), (4) DOX+Nrf2 siRNA treated, (5) DOX +ORM1 treated, and (6) DOX+ORM1+Nrf2 siRNA treated. After treatment, the cells were collected for further analyses.

2.3. Echocardiography. Seven days after the first DOX injection, the mice were anesthetized with $1.5 \%$ isoflurane to stabilize the heart rate at $400-500 \mathrm{bpm}$. The size and function of the hearts were measured by M-mode echocardiography using the ms-400 linear transducer echocardiography system (Visual Sonics Vevo 2100, Toronto, CA). All measurements are the average of five consecutive heartbeat cycles.

2.4. Measurement of Plasma Levels of Lactate Dehydrogenase $(\mathrm{LDH})$ and Creatine Kinase Isoenzyme (CKMB). Blood samples were taken from mice in the control and DOX groups and poured into EDTA tubes to prevent clotting. Plasma was separated by centrifugation. $\mathrm{LDH}$ and $\mathrm{CKMB}$ levels in plasma were determined using the standard enzyme-linked immunosorbent assay (ELISA) kit (Roche, Switzerland) and presented in U/l. All procedures were performed according to the manufacturer's instructions.

2.5. Wheat Germ Agglutinin (WGA) Staining. Hearts were collected from five mice in the control and DOX groups, respectively, and then fresh-frozen heart tissues were stained with WGA (Sigma, USA) according to the manufacturer's instructions (200 cross-sectional areas per mouse). Fluorescence images were examined using a Zeiss Axio Imager 2 microscope (Zeiss, Germany). 
2.6. Terminal Deoxynucleotidyl Transferase dUTP Nick End Labeling (TUNEL) Assay. Hearts were collected from five mice in the control and DOX groups, respectively. Frozen heart tissues were cut into $5 \mu \mathrm{m}$ thick sections, and H9c2 cells were cultured on cover glass. H9c2 cells were transfected with ORM1 virus, Nrf2 siRNA, or the corresponding control. After $48 \mathrm{~h}$ of transfection, cells were stimulated with DOX for $24 \mathrm{~h}$ and fixed with $4 \%$ paraformaldehyde. Apoptosis in myocardial tissues was analyzed using a TUNEL staining kit (Roche, Switzerland), and H9c2 cells were analyzed using a different TUNEL staining kit (Abcam, UK) according to the manufacturer's instructions. Fluorescence images were examined using a Zeiss Axio Imager 2 microscope (Zeiss, Germany).

2.7. Liquid Chromatography-Tandem Mass Spectrometry ( $L C / M S)$. The hearts were collected from mice in the control and DOX groups, and three mice from each group were sent to Bio Miao Biological and processed according to the corresponding procedure. The peptide mixtures in each group of samples were labeled with different isobaric tags for relative and absolute quantification (iTRAQ) reagents; equal amounts of the labeled peptides in each sample were prepared. Finally, LC/MS detection and analysis were performed.

2.8. Immunohistochemical Staining. Mouse hearts were fixed in $4 \%$ paraformaldehyde ( $\mathrm{pH} 7.4$ ) overnight, embedded in paraffin, and continuously sectioned at a thickness of $5 \mu \mathrm{m}$. For dewaxing, sections were sealed with phosphate-buffered saline (PBS) containing 5\% normal target serum and $1 \%$ bovine serum albumin (BSA) and then incubated overnight at $4^{\circ} \mathrm{C}$ under humidified conditions with anti-ORM1 (Abcam, UK), anti-Nrf2 (Abcam, UK), anti-HO-1 (Abcam, UK), anti-4-HNE (Abcam, UK), and anticleaved caspase-3 (Cell Signaling Technology, USA). A routine histological examination was performed using light microscopy. Images were examined with a Zeiss Axio Imager 2 microscope (Zeiss, Germany).

2.9. Analysis of Cell Viability. Cell survival rate was determined using the Cell Counting Kit-8 (CCK-8, Beyotime, China) according to the manufacturer's instructions. H9c2 cells $\left(1 \times 10^{4}\right)$ were seeded onto 96-well plates and subjected to the treatments described above. CCK- 8 solution $(10 \mu \mathrm{L})$ was added to $100 \mu \mathrm{L}$ of culture medium in each well and incubated for $2 \mathrm{~h}$ at $37^{\circ} \mathrm{C}$, before the optical density (OD) was measured at $450 \mathrm{~nm}$ with a Thermo Multiskan FC microplate reader (Thermo, USA). Cell survival rates were expressed as the ratio of the OD value of the experimental well to that of the control well. Each treatment was performed six times. Cell survival rates were expressed as the ratio of the OD value of the experimental well to that of the control well.

2.10. Malondialdehyde (MDA) Determination. To analyze oxidative stress parameters, we examined the MDA content. H9c2 cells $\left(1 \times 10^{4}\right)$ were seeded onto 96-well plates and subjected to the treatments described above. Blood samples were collected from mice in the control and DOX groups.
We determined the MDA content using a commercially available MDA detection kit (Beyotime, China) according to the manufacturer's instructions.

\subsection{Dichlorodihydrofluorescein Diacetate (DCFH-DA)} Assay. The production of ROS was established using a DCFH-DA staining kit (Beyotime, China). H9c2 cells were seeded onto 96-well plates and subjected to the treatments described above, then incubated with $10 \mu \mathrm{M}$ DCFH-DA for $30 \mathrm{~min}$ in the dark at $37^{\circ} \mathrm{C}$. Fluorescence intensities were immediately measured by spectrophotometry using a microplate reader; the excitation and emission wavelengths were 488 and $522 \mathrm{~nm}$, respectively. The fluorescence intensity of the control group was set to $100 \%$. The accumulation of ROS in H9c2 cells was analyzed by confocal microscopy (red staining), and the fluorescence intensity of DCFH-DA was quantified using the Image Pro Plus software (Media Cybernetics Inc., USA).

2.12. Polymerase Chain Reaction (PCR) Analysis. Trizol (Invitrogen, USA) was used to extract total RNA from heart tissues and cells, and the concentration and purity of RNA were determined by spectrophotometry. The PrimeScript RT Kit (Takara, Japan) was used to synthesize cDNA according to the manufacturer's instructions. Quantitative PCR was performed using the CFX96 Real-Time System (Bio$\mathrm{Rad}$ ) to detect differences in gene expression. Relative gene expression of each protein of interest was calculated using the $2^{-\triangle \Delta C T}$ method and normalized to GAPDH expression. All reactions were performed in triplicate, and the specificity was monitored by melting curve analysis. The primers were purchased from RiboBio (China) (see S-Fig4 for the PCR primers used).

2.13. Western Blot Analysis. After collecting cells and heart samples from the different treatment groups and extracting proteins, the protein concentrations were determined using a bicinchoninic acid (BCA) protein assay (Thermo Scientific, USA). The samples from each group were separated by sodium dodecyl sulfate polyacrylamide gel electrophoresis (SDS-PAGE) and transferred to a polyvinylidene fluoride (PVDF) membrane. The membrane was blocked by incubation with 5\% skimmed milk (containing $0.1 \%$ Tween-20) for $2 \mathrm{~h}$ at room temperature. After incubation, the membrane was further incubated with the primary antibodies antiORM1 (Abcam, UK), anti-Nrf2 (Abcam, UK), anti-HO-1 (Abcam, UK), anti-4-HNE (Abcam, UK), and anticleaved caspase-3 (Cell Signaling Technology, USA). Next, the membrane was washed in Tris-buffered saline Tween (TBST) and incubated with the corresponding secondary antibody (Abcam; at a dilution of $1: 5000$ ) for $2 \mathrm{~h}$ at room temperature. The Amersham Imager 680 (GE, USA) was used to detect the bands, and the signal was quantified.

2.14. Statistical Analysis. The data are expressed as the mean \pm standard error of the mean (SEM). Data were analyzed using the SPSS 19.0 statistical software (SPSS Inc., USA). Differences between two groups were calculated using the $t$-test, and one-way analysis of variance (ANOVA) with Tukey's post hoc test was used when comparing multiple 


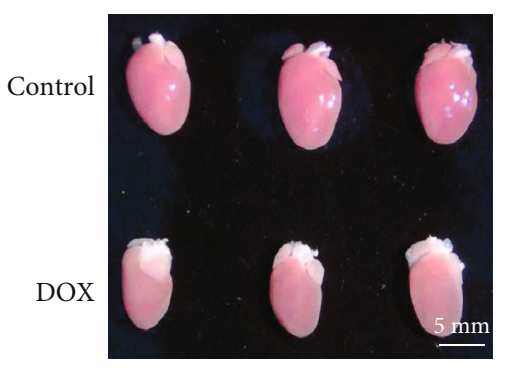

(a)

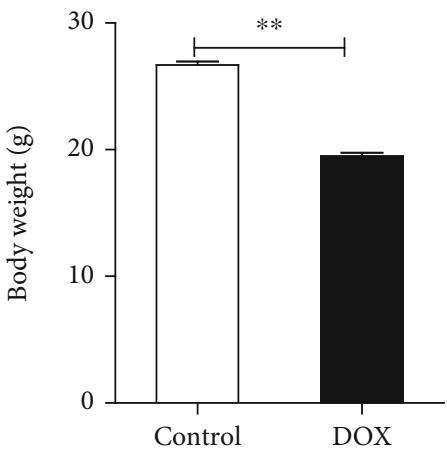

(b)

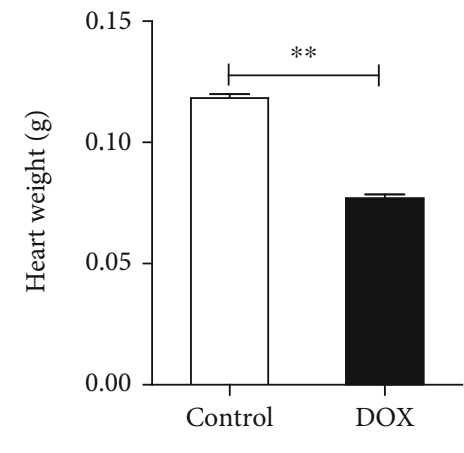

(c)

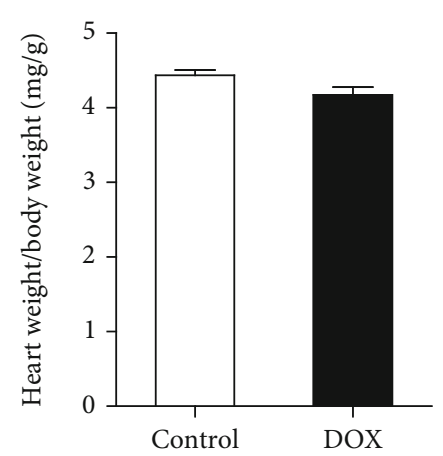

(d)

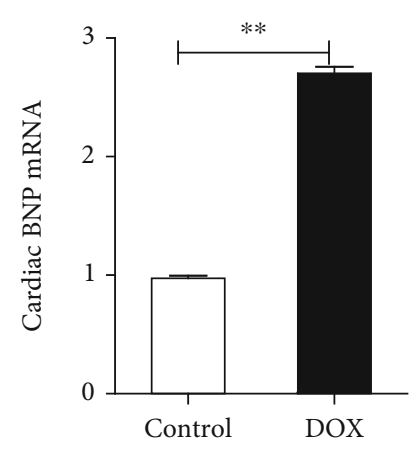

WGA

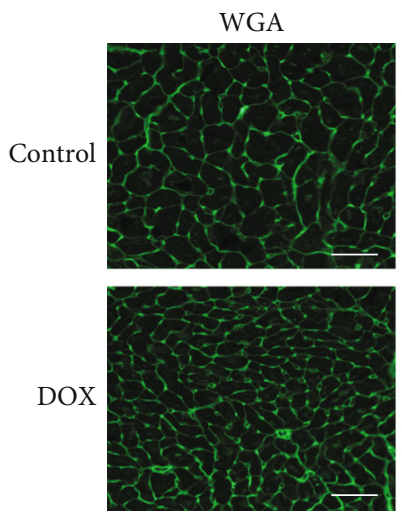

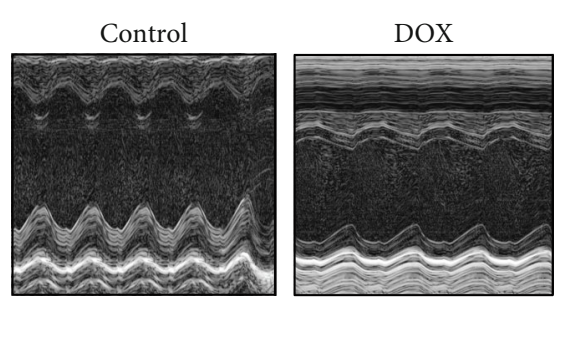

(e)

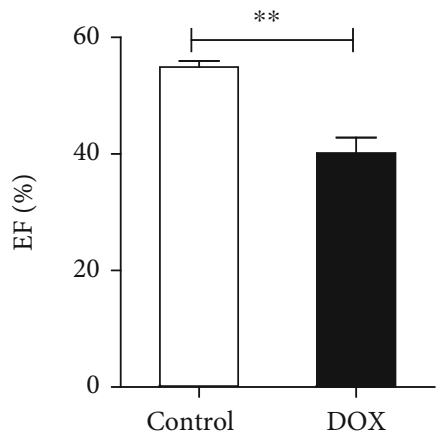

(g)

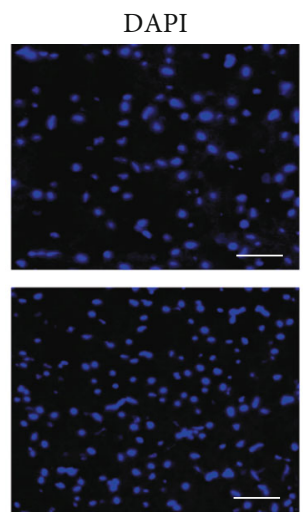

(i)

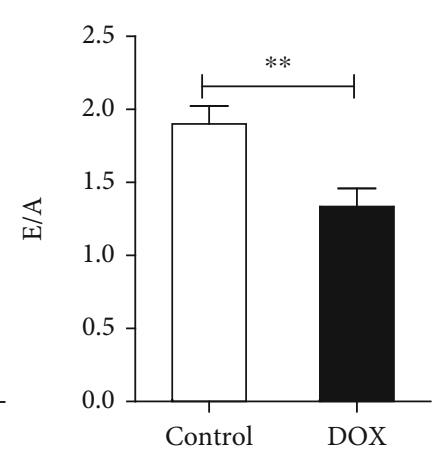

Merge
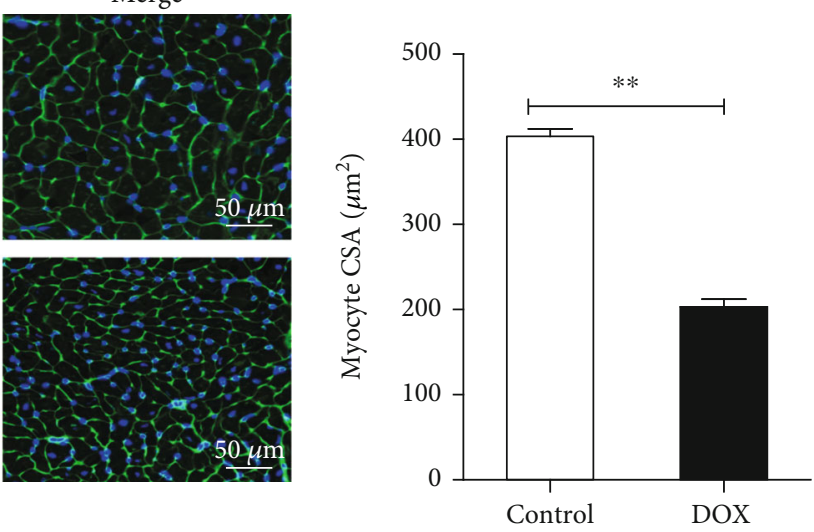

(j)

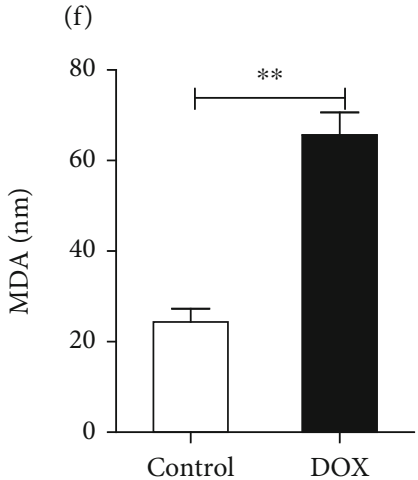

(h)

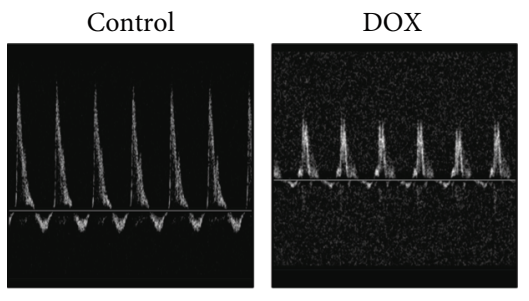



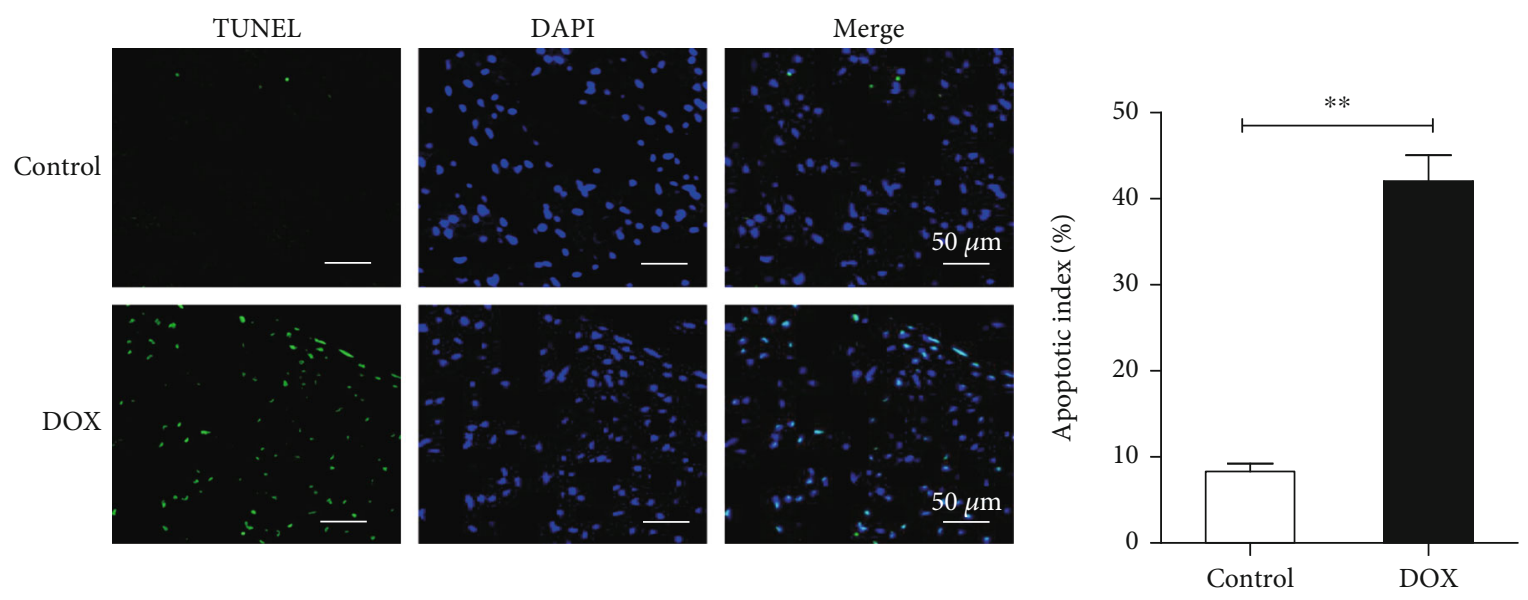

(k)

(l)

Figure 1: Effects of doxorubicin (DOX) on C57BL/6 mice. (a) General pictures of the heart. (b) Body weight. (c) Heart weight. (d) Heart weight/body weight. (e, f) Systolic and diastolic functions in both groups $(n=20)$. (g) B-type natriuretic peptide (BNP) levels in mice detected using polymerase chain reaction (PCR) $(n=10)$. (h) Malondialdehyde (MDA) levels in both groups $(n=10)$. (i, j) Myocardial cell size evaluated using wheat germ agglutinin (WGA) staining $(n=5)$. $(\mathrm{k}, \mathrm{l})$ Terminal deoxynucleotidyl transferase dUTP nick end labeling (TUNEL) staining $(n=5)$. Data are expressed as the mean \pm standard error of the mean $(\mathrm{SEM}) .{ }^{* *} P<0.01$.

groups. Differences with a $P$ value $<0.05$ were considered significant.

\section{Results}

3.1. DOX Caused Cardiac Dysfunction and Heart Injury in C57BL/6 Mice. All experimental mice were weighed to determine weight loss during each treatment. The results showed that the weight of DOX-treated mice was higher than that of the control group. After sacrificing, it was found that the hearts of DOX-treated mice were significantly atrophic, and the heart weight was significantly reduced compared to that of the normal group, indicating DOX-induced cardiac atrophy (Figures 1(a)-1(d)). DOX caused acute cardiac dysfunction in male mice, leading to a significant decrease in systolic and diastolic functions relative to those of the control group (Figures 1(e) and 1(f), Supplementary Figure 1a). To determine the levels of myocardial injury caused by DOX, we tested the serum $\mathrm{LDH}$ and CKMB levels and found that the difference was statistically significant between the DOXtreated and control groups (Supplementary Figure 1b-c). To evaluate cell damage induced by DOX, we measured the B-type natriuretic peptide (BNP) and A-type natriuretic peptide(ANP) levels $7 \mathrm{~d}$ after DOX administration and found that the mRNA levels of BNP and ANP were significantly increased in DOX-treated mice, indicating obvious heart failure (Figure 1(g), Supplementary Figure 1d).

According to previous studies, one of the main mechanisms of DOX-mediated myocardial injury is oxidative stress injury. Therefore, we examined the serum MDA levels in the mice and found that the level in the DOX group was significantly higher than that in the control group (Figure $1(\mathrm{~h})$ ). To evaluate the DOX-induced damage to cardiomyocytes, we stained murine heart sections with WGA and found that cardiomyocytes from DOX-treated mice were significantly reduced, which is in accordance with the observed atrophy (Figures 1(i) and 1(j)). To evaluate the level of cardiomyocyte apoptosis, we conducted TUNEL staining of the myocardial tissues of the mice. The results showed that compared with the control group, the DOX group exhibited a significantly higher apoptosis index (Figures 1(k) and 1(l)).

\subsection{DOX Treatment Evoked the Downregulation of ORM1} and Upregulation of Oxidative Stress and Apoptosis in Murine Myocardial Tissue. First, we collected the hearts from control and DOX-treated mice and performed LC/MS analysis. The heat map shows differentially expressed genes between the cardiac tissues of control and DOX-treated mice. Low expression is depicted in green, and high expression is depicted in red. We found that ORM1 levels were significantly decreased in DOX-treated mice (Figure 2(a)). We then examined the mRNA levels of ORM1, Nrf2, and HO-1 in vivo. Compared with those in the control group, the mRNA levels of ORM1, Nrf2, and HO-1 were significantly reduced in the DOX group (Figure 2(b)). The protein levels of ORM1, Nrf2, and HO-1 were also significantly reduced in the DOX group, which was consistent with the effect on the mRNA levels (Figures 2(c) and 2(d)). In addition, 4$\mathrm{HNE}$ is the downstream molecule of HO-1, which can cause extensive oxidative damage and cell apoptosis, and cleaved caspase- 3 is a marker of cell apoptosis. Therefore, we chose to examine 4-HNE and cleaved caspase-3 to evaluate the effects of DOX on the level of oxidative stress and cell apoptosis. Compared with those in the control group, the protein levels of 4-HNE and cleaved caspase-3 were significantly increased in the DOX group (Figures 2(c) and 2(d)). The results of immunohistochemical staining of the myocardia from DOX-treated and control mice confirmed the above findings (Figures 2(e) and 2(f)). 


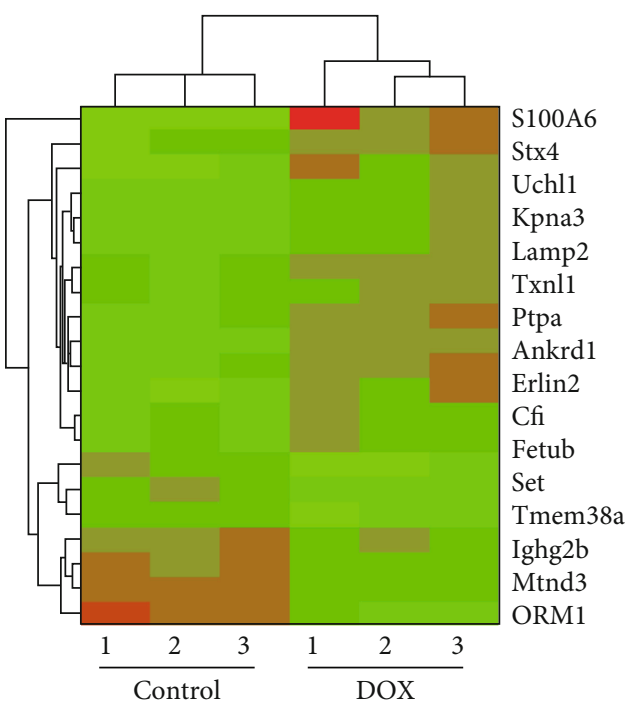

(a)

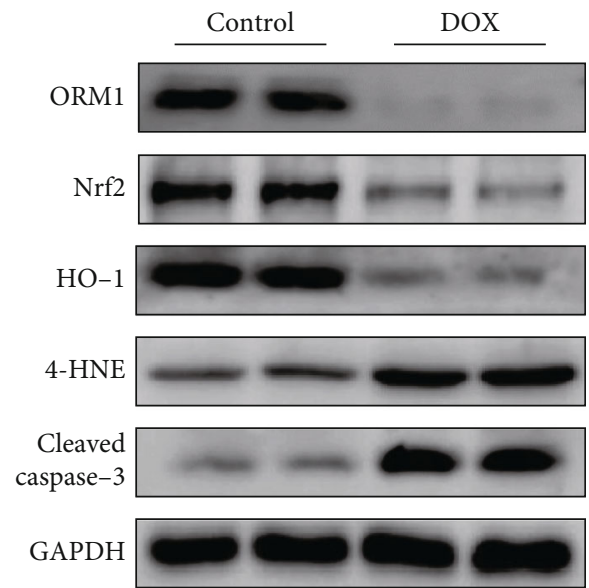

(c)

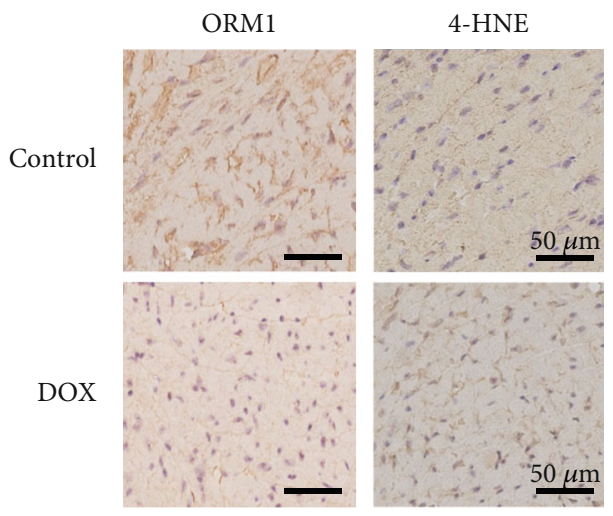

(e)

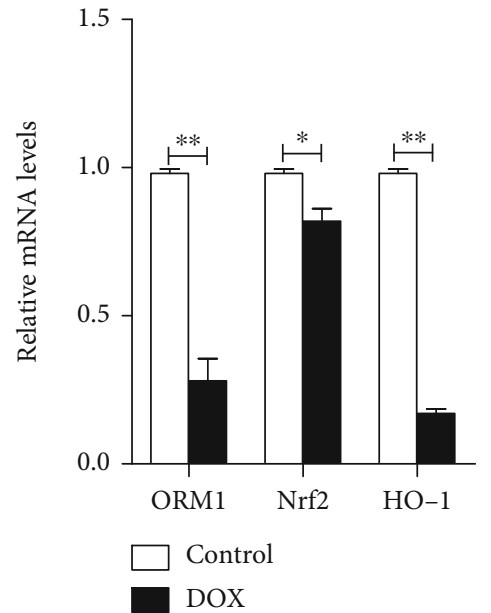

(b)

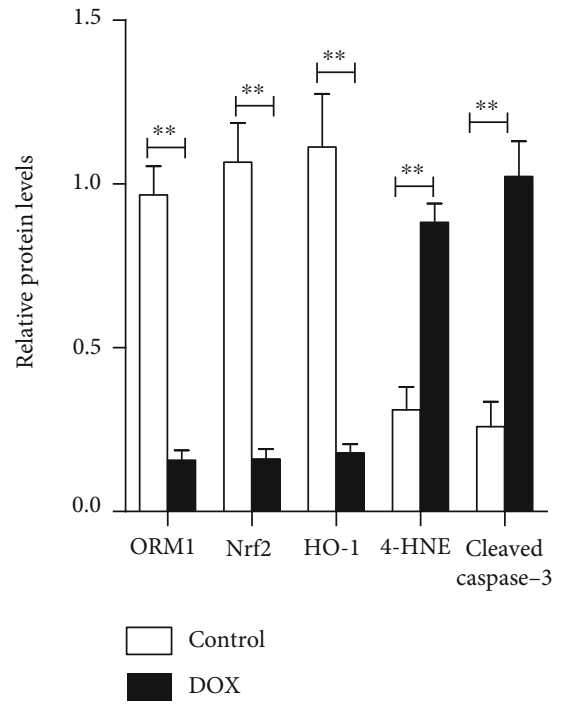

(d)

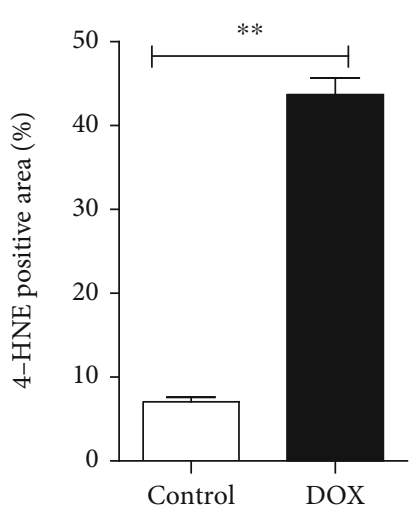

(f)

Figure 2: Continued. 


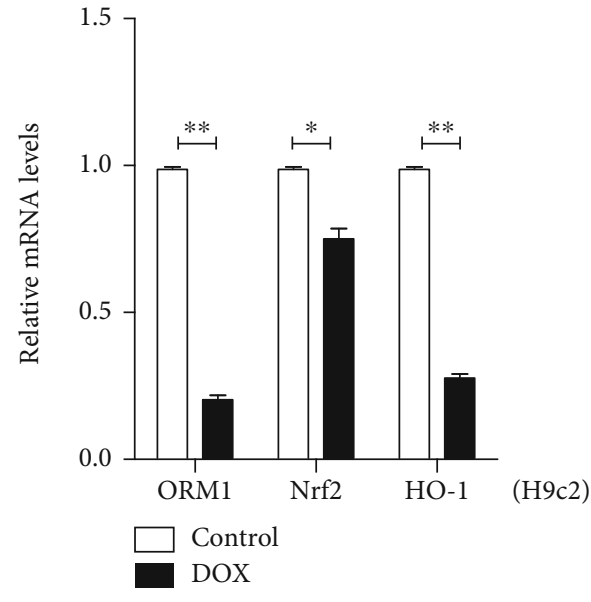

(g)

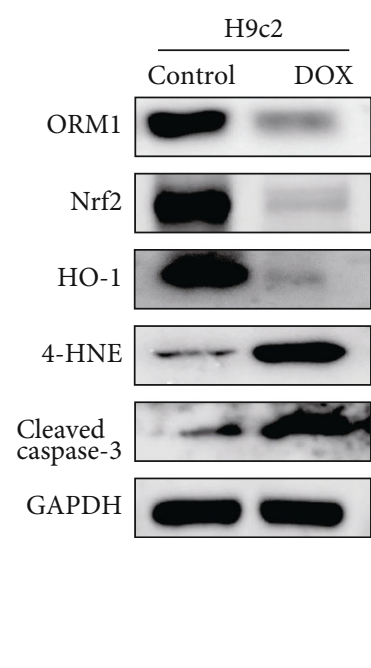

(h)

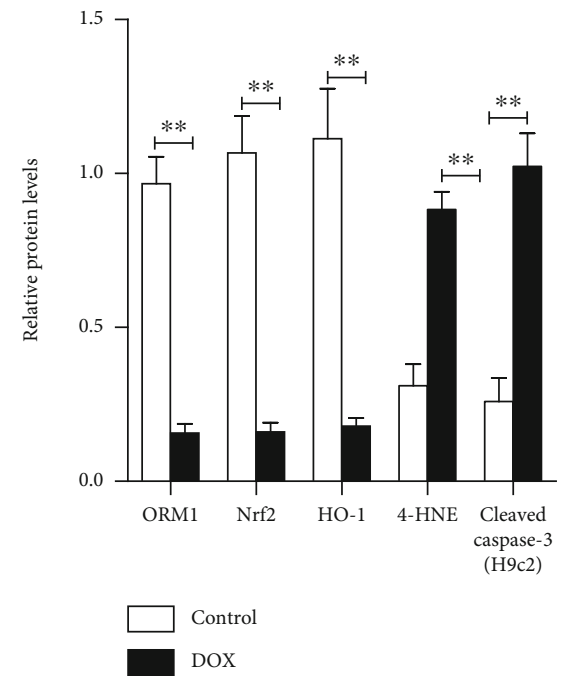

(i)

FIGURE 2: Downregulation of ORM1 and upregulation of oxidative stress and apoptosis in the DOX-induced cardiomyopathy model in vitro and in vivo. (a) Differentially expressed candidate proteins in control and DOX-treated mice analyzed using LC/MS technology. Low expression is depicted in green, and high expression is depicted in red. (b) mRNA levels of ORM1, Nrf2, and HO-1 in the hearts of mice in the DOX-treated groups $(n=6)$. (c, d) Western blot analysis of ORM1, Nrf2, HO-1, 4-HNE, and cleaved caspase-3 ( $n=6)$. (e, f) Immunohistochemical staining images of ORM1- and 4-HNE-stained heart sections from control and DOX-treated mice $(n=6)$. (g) mRNA levels of ORM1, Nrf2, and HO-1 in DOX-treated H9c2 cells $(10 \mu \mathrm{M}$ and $24 \mathrm{~h})$ and the control group $(n=3)$. (h, i) Western blot analysis of ORM1, Nrf2, HO-1, 4-HNE, and cleaved caspase-3 in DOX-treated H9c2 cells (10 $\mu \mathrm{M}$ and $24 \mathrm{~h})$ and the control group ( $n=3)$. Data are expressed as the mean \pm standard error of the mean $(\mathrm{SEM}) .{ }^{* *} P<0.01$ and ${ }^{*} P<0.05$.

\subsection{DOX Treatment Produced the Downregulation of ORM1} and Upregulation of Oxidative Stress and Apoptosis in H9c2 Cells. In H9c2 cells, different concentrations of $\operatorname{DOX}(0,2$, 5 , and $10 \mu \mathrm{M}$ ) were used to examine the effect of DOX on cell viability. The results showed that DOX significantly reduced cell viability in a concentration-dependent manner; the effect was most obvious when the concentration was $10 \mu \mathrm{M}$ (Supplementary Figure 2). Therefore, $10 \mu \mathrm{M}$ DOX was used in subsequent experiments.

The mRNA and protein levels of ORM1, Nrf2, and HO-1 in $\mathrm{H} 9 \mathrm{c} 2$ cells treated with DOX were significantly decreased (Figures 2(g)-2(i)). In addition, the levels of 4-HNE and cleaved caspase- 3 were significantly increased in $\mathrm{H} 9 \mathrm{c} 2$ cells after DOX stimulation (Figures 2(h) and 2(i)). These results were consistent with the in vivo results and indicated that decreased levels of ORM1 might play an important role in DOX-induced cardiotoxicity.

3.4. ORM1 Reduced DOX-Induced Oxidative Stress and Apoptosis in H9c2 Cells. To explore the protective effect of ORM1 against DOX in $\mathrm{H} 9 \mathrm{c} 2$ cells in vitro, the effect of ORM1 on the viability of H9c2 cells was measured. A concentration of ORM1 below 100 MOI produced no cytotoxic effects. However, a higher concentration slightly reduced the viability of $\mathrm{H} 9 \mathrm{c} 2$ cells (Supplementary Figure 3a). Cotreatment of $10 \mu \mathrm{M}$ DOX and either $10,30,50$, or 100 MOI ORM1 caused a concentration-dependent increase in cell viability compared to that of the control group (Supplementary Figure 3b). Further experiments were carried out using $10 \mu \mathrm{M}$ DOX.
Next, we more closely examined the effects of 100 MOI ORM1 on H9c2 cells treated with DOX. Western blot analysis demonstrated that the addition of exogenous ORM1 reduced the oxidative level and apoptosis index in cardiomyocytes, reversing the DOX-induced effects (Figures 3(a) and 3(b)). The CCK-8 assay was utilized to further examine the protective effects of ORM1 in H9c2 cells. The results showed that the cell survival rate significantly increased in DOX+ORM1-treated cells compared with that in cells treated with DOX only (Figure 3(c)).

MDA is a parameter of oxidative stress, and its content in cells was significantly upregulated by DOX (Figure 3(d)). Under various pathological conditions, the initiation of oxidative stress and the overproduction of ROS play a key role in the development of cardiac dysfunction. A DCFH-DA assay was utilized to analyze the production of cellular ROS, and the fluorescence intensity increased with the production of reactive metabolites. The combination of ORM1 and DOX effectively inhibited ROS production (Figures 3(e) and 3(f)). A subsequent TUNEL assay of $\mathrm{H} 9 \mathrm{c} 2$ cells showed that DOX significantly increased apoptosis, while ORM1 produced the opposite effect (Figures $3(\mathrm{~g})$ and $3(\mathrm{~h})$ ).

3.5. Nrf2 Knockdown Reversed the Protective Effects of ORM1 in DOX-Treated H9c2 Cells. To study the role of the Nrf2mediated transcription network in the DOX-induced cardioprotective effects of ORM1, Nrf2 was knocked down by specific Nrf2 siRNA. Western blot analysis showed that Nrf2 knockdown did not affect the ORM1 level, it downregulated 


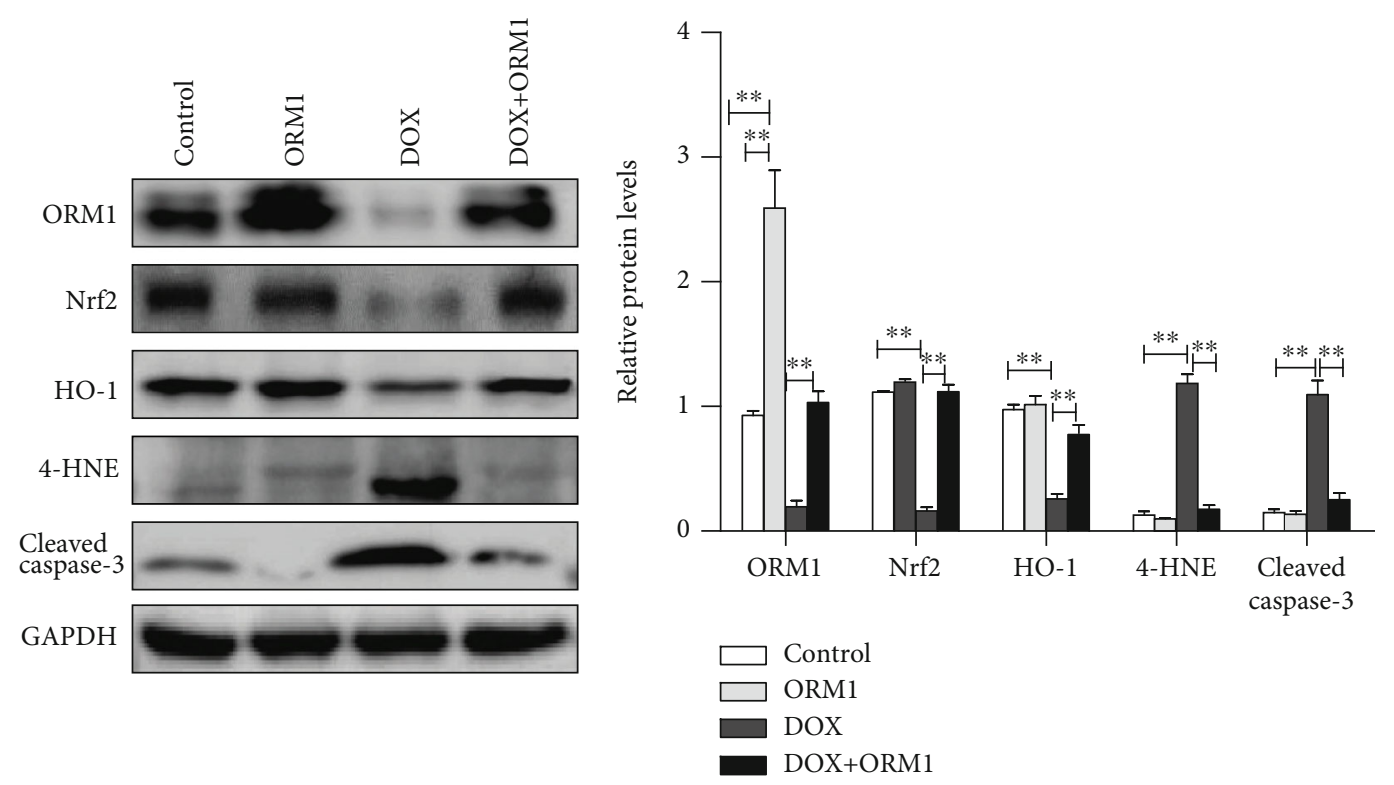

(a)

(b)

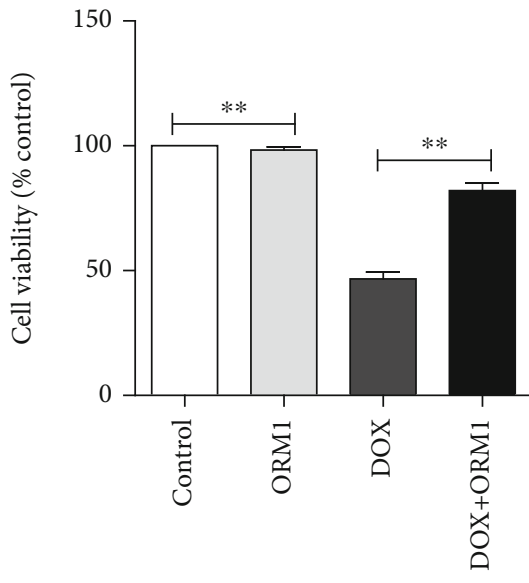

(c)

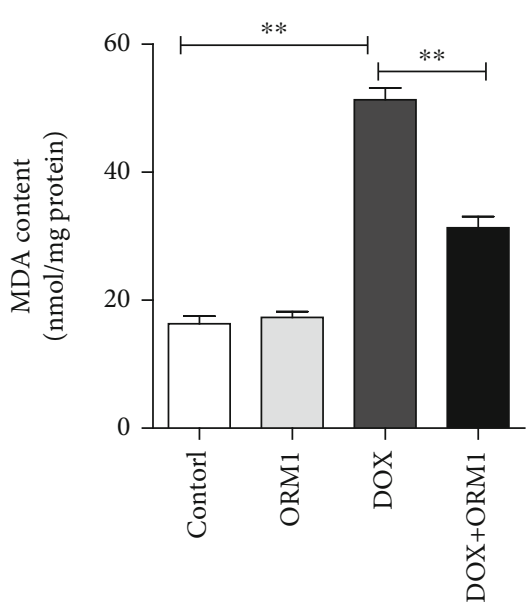

(d)

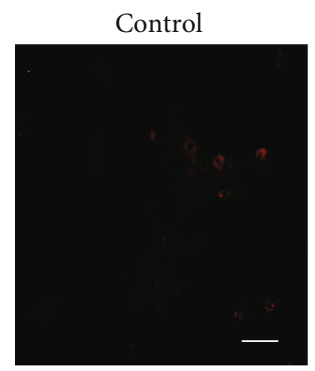

ORM1

DOX
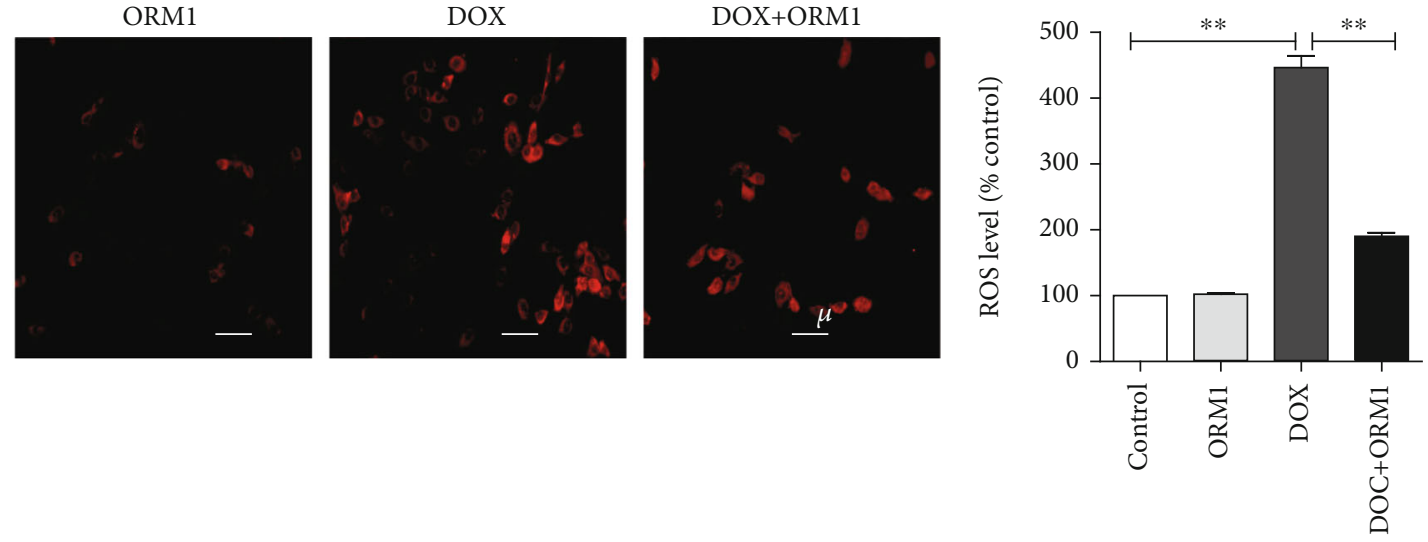

(e)

(f)

Figure 3: Continued. 

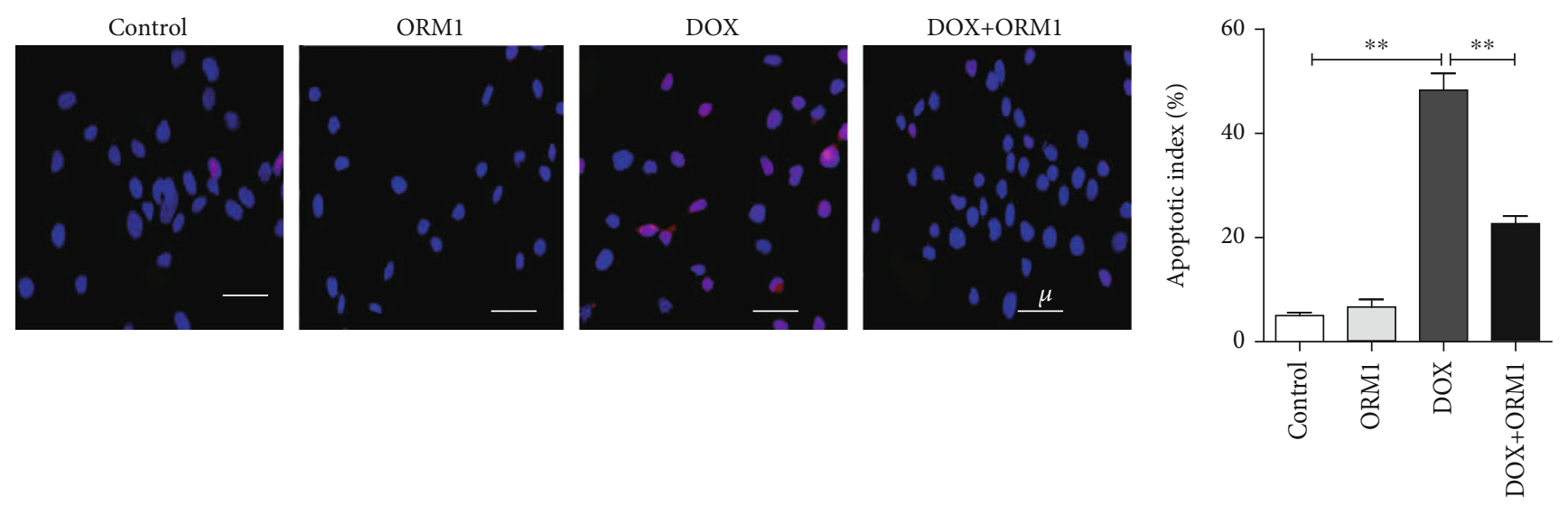

(g)

(h)

FIGURE 3: ORM1 reduces doxorubicin- (DOX-) induced oxidative stress and apoptosis in H9c2 cells. (a, b) Western blot analysis of ORM1, Nrf2, HO-1, 4-HNE, and cleaved caspase-3. (c) Cell survival rate analysis using the Cell Counting Kit 8 (CCK-8). Cell survival rate is expressed as the optical density (OD) value (\% control). (d) Cellular malondialdehyde (MDA) content. (e, f) Fluorescence image (red fluorescence) of reactive oxygen species (ROS) measured using dichlorodihydrofluorescein diacetate (DCFH-DA). (g, h) Terminal deoxynucleotidyl transferase dUTP nick end labeling (TUNEL) staining images with calculated apoptosis indices. Data are expressed as the mean \pm standard error of the mean $(\mathrm{SEM}) ; n=6 .{ }^{* *} P<0.01$.

HO-1 expression and upregulated 4-HNE and cleaved caspase- 3 levels in the DOX+ORM1+Nrf2 siRNA group compared to the corresponding levels in the DOX+ORM1 group (Figures 4(a) and 4(b)). CCK-8 analysis showed that compared with cells expressing Nrf2, Nrf2-knockdown cells exhibited an aggravated reduction of cell viability in DOX group, while silencing Nrf2 significantly reduced cell viability in the DOX+ORM1+Nrf2 siRNA group compared to the corresponding levels in the DOX+ORM1 group in H9c2 cells (Figure 4(c)). Similarly, silencing Nrf2 significantly promoted the production of ROS and MDA in DOX-treated $\mathrm{H} 9 \mathrm{c} 2$ cells, and Nrf2 knockdown reversed the protection of ORM1 in DOX-treated H9c2 cells. In conclusion, inhibition of Nrf2 activation reversed the antioxidative effects of ORM1 against DOX-induced myocardial toxicity in $\mathrm{H} 9 \mathrm{c} 2$ cells (Figures 4(d)-4(f)). TUNEL analysis showed that Nrf2 siRNA reversed the protective effects of ORM1 in DOXtreated H9c2 cells (Figures 4(g) and 4(h)). These findings confirmed that the Nrf2 pathway was involved in the protective function of ORM1 in cardiomyocytes affected by DOXinduced oxidative stress and apoptosis.

\section{Discussion}

DOX has been used for more than half a century as an anticancer drug and is the cornerstone of chemotherapy in children and adults; however, its cardiotoxicity poses a serious threat. Accumulating evidence shows that oxidative stress and cell apoptosis play key roles in the pathogenesis of DOX-induced myocardial injury $[23,24]$, which is caused by the elevated production of ROS and 4-HNE (the final product of lipid peroxidation $[25,26])$ and leads to cardiomyocyte apoptosis.

In our study, LC/MS technology was used to identify and quantify candidate proteins that are differentially expressed in response to DOX-induced cardiotoxicity. We found that
ORM1 levels were significantly decreased, and analyses of in vivo and in vitro DOX-induced myocardial injury models confirmed this result. We also found that the levels of oxidative stress and apoptosis were increased and that ORM1 inhibited oxidative stress and apoptosis in H9C2 cells via Nrf2 signaling. Many studies have shown that various molecular mechanisms and signaling pathways can regulate oxidative stress and cardiomyocyte apoptosis; for example, activation of the Nrf2/HO-1 pathway can affect DOXinduced myocardial injury [19-21]; our study produced consistent findings and showed that the $\mathrm{Nrf} 2 / \mathrm{HO}-1$ pathway also participates in the antiapoptotic action of ORM1 in DOXtreated $\mathrm{H} 9 \mathrm{c} 2$ cells.

$\mathrm{Nrf} 2$ is the master regulator of cellular redox homeostasis and is also involved in maintaining mitochondrial redox homeostasis by providing reduced forms of glutathione (GSH) and mitochondrial antioxidant enzymes such as GSH peroxidase, superoxide dismutase, and peroxiredoxin. Nrf2 deficiency results in impaired mitochondrial fatty acid oxidation, respiration, and adenosine triphosphate (ATP) production. Recent studies have shown that Nrf2 also affects mitochondrial function in cardiomyocyte regeneration and neural stem/progenitor cell survival [27-29]. However, the function of Nrf2 in myocardial injury caused by DOX is not completely understood. In future studies, we intend to further analyze the function of Nrf2 in the mitochondria of DOX-treated mice.

The addition of exogenous ORM1 reduced both the oxidative stress and apoptosis caused by DOX. DOX-induced oxidative stress was significantly reversed when $\mathrm{H} 9 \mathrm{c} 2$ cells were treated with a combination of ORM1 and DOX, confirming the antioxidative power of ORM1. Cardiomyocyte apoptosis is another hallmark of acute DOX-induced cardiotoxicity, and ORM1 reportedly exhibits protective effects against apoptosis under various pathogenic conditions [12-14]. The results from the TUNEL assay confirmed the antiapoptotic effect of ORM1, as the protein significantly inhibited DOX- 

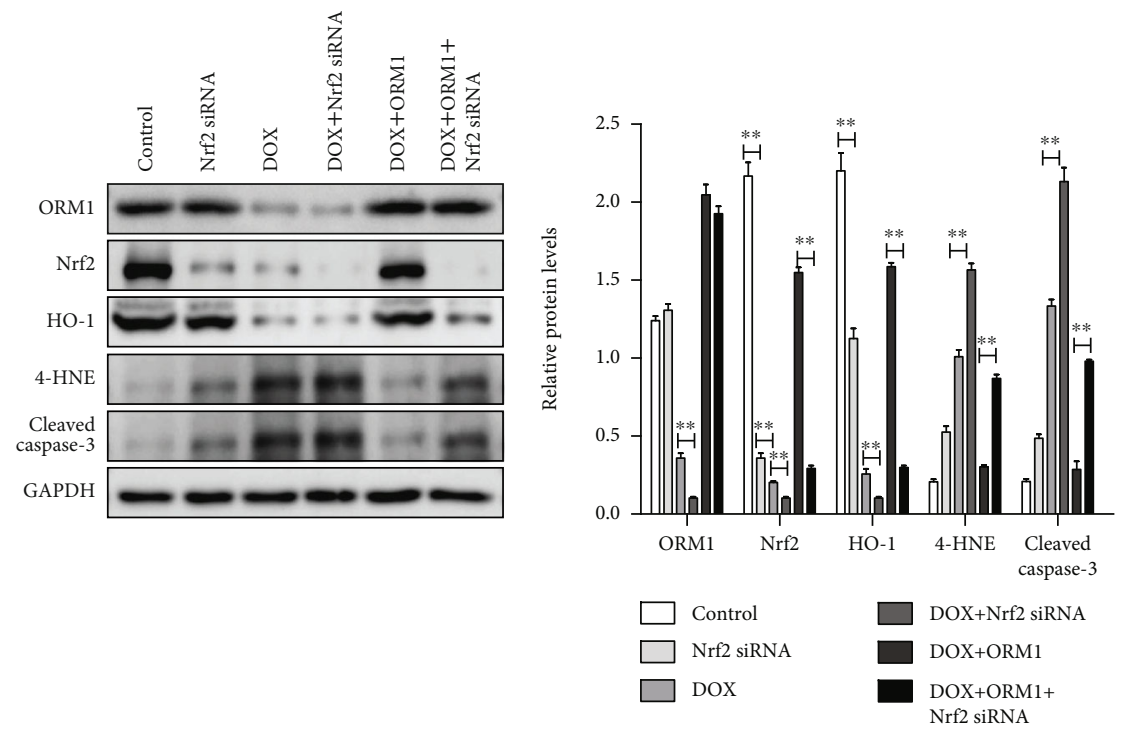

(a)

(b)

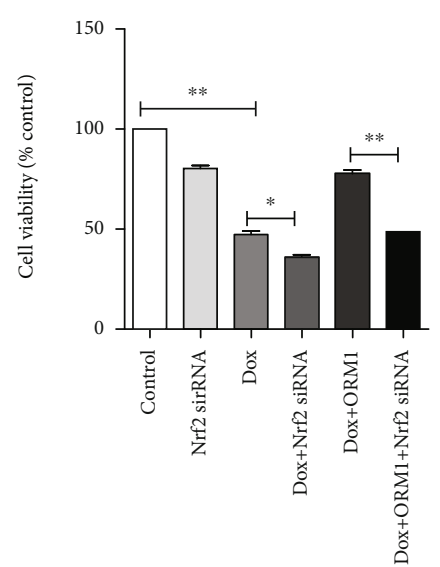

(c)

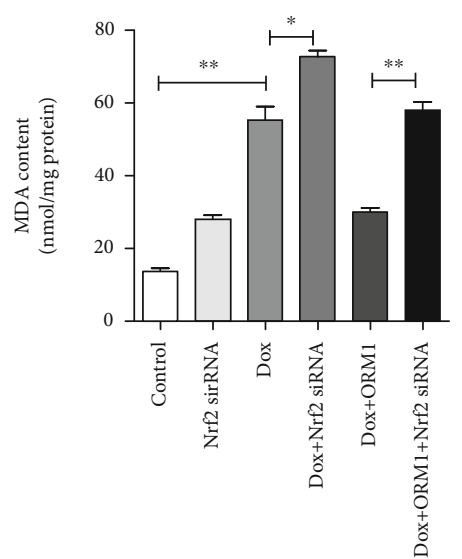

(d)

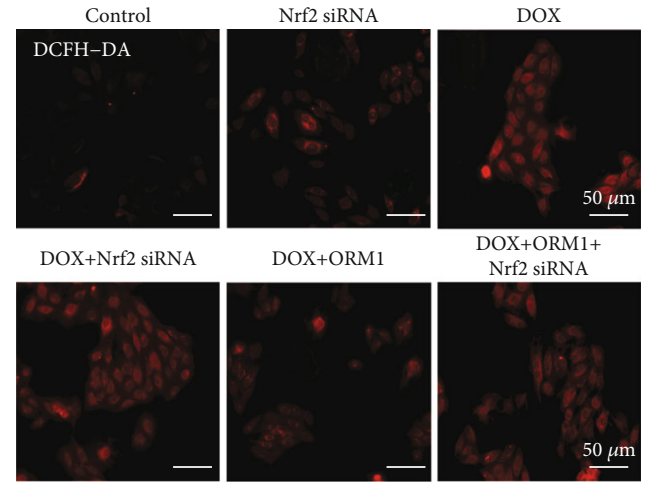

(e)

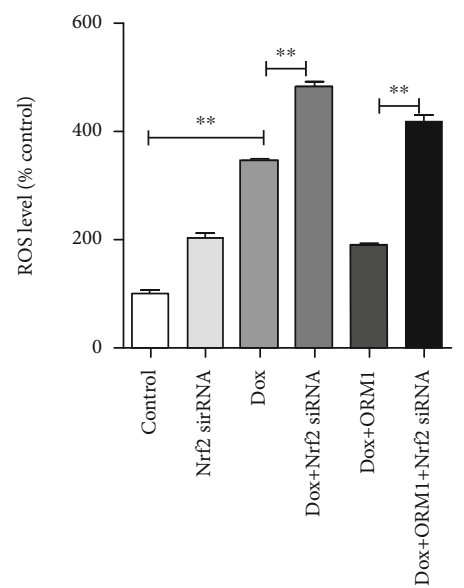

(f)

FIgURE 4: Continued. 


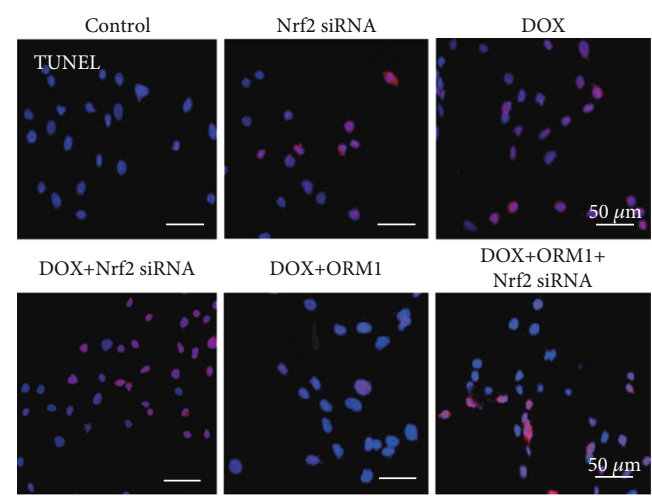

(g)

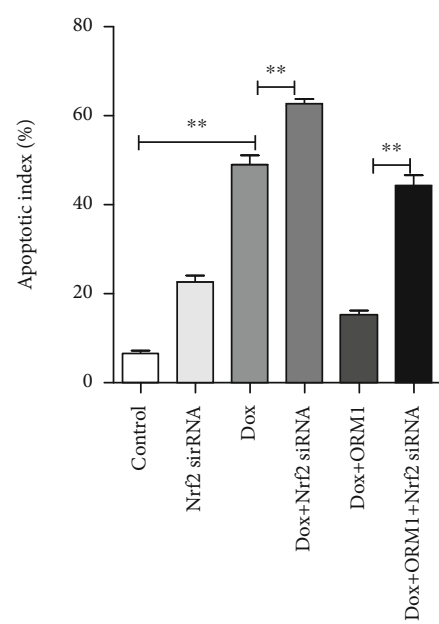

(h)

FIGURE 4: Nrf2 knockdown reverses the protective effects of ORM1 in doxorubicin- (DOX-) treated H9c2 cells. (a, b) Western blot analysis of ORM1, Nrf2, HO-1, 4-HNE, and cleaved caspase-3. (c) Cell survival analysis using the Cell Counting Kit 8 (CCK-8). (d) Cellular malondialdehyde (MDA) content. (e, f) Fluorescence image (red fluorescence) of reactive oxygen species (ROS) measured using dichlorodihydrofluorescein diacetate (DCFH-DA). (g, h) Terminal deoxynucleotidyl transferase dUTP nick end labeling (TUNEL) staining images with calculated apoptosis indices. Data are expressed as the mean \pm standard error of the mean $(\mathrm{SEM}) ; n=6$. ${ }^{* *} P<0.01$ and ${ }^{*} P<0.05$.

induced apoptosis in vitro. Nrf2 silencing negated the protective effects of ORM1 against DOX-induced cardiomyocyte injury, suggesting that ORM1 regulates DOX-induced cardiomyocyte oxidation and apoptosis by upregulating Nrf2/HO-1 signaling and other important antioxidative Nrf2-related pathways that influence cell apoptosis $[30,31]$.

There were several limitations to this study. First, the effects of ORM1 on DOX-induced oxidative stress and apoptosis in cardiomyocytes were only examined in vitro. ORM1 cardiac-specific transgenic mice or knockout mice will be considered in future studies to clarify the key role of ORM1 in vivo. Moreover, it is not clear whether there was an Nrf2 binding site in the promoter region of the ORM1 gene. Chromatin immunoprecipitation and luciferase reporter gene assays will be used to clarify the details of the relationship between ORM1 and Nrf2.

\section{Conclusions}

In summary, our study showed that ORM1 could attenuate DOX-induced oxidative stress and apoptosis in cardiomyocytes via Nrf2 signaling, suggesting that ORM1 might be an effective therapeutic target for the treatment of DOXinduced cardiotoxicity. This finding provides novel insights that may contribute to significant advances in the field of oncology.

\section{Data Availability}

The data used in this study are available upon request from the corresponding author.

\section{Conflicts of Interest}

The authors declare that there is no conflict of interest regarding the publication of this paper.

\section{Acknowledgments}

This work was supported by the National Natural Science Foundation of China (81670276, 81870553, 81770303, and 91739118), Natural Science Foundation of Liaoning Province (20180550368), Science and Technology Project of Shenyang (19-112-4-056), and Military Science and Technology Youth Talents Entrustment Project (17JCJQ-QT-028).

\section{Supplementary Materials}

Fig. S1: DOX causes heart failure on C57BL/6 mice. (a) The level of FS valve $(n=20)$. (b) Serum LDH $(n=10)$. (c) Serum CK/MB $(n=10)$. (d) Relative ANP mRNA levels of hearts $(n=10)$. Data are expressed as the mean \pm standard error of the mean $(\mathrm{SEM}) ;{ }^{* *} P<0.01$. Fig S2: DOX causes a dosedependent inhibition of cell viability in H9c2 cells $(n=3)$. Data are expressed as the mean \pm standard error of the mean $(\mathrm{SEM}) ;{ }^{*} P<0.05$ and ${ }^{* *} P<0.01$. Fig. S3: ORM1 upregulated cell viability in DOX-induced cardiomyocytes. (a) The cell viability with different dose ORM1 $(n=3)$. (b) ORM1 upregulated cell viability in DOX-induced cardiomyocytes $(n=3)$. Data are expressed as the mean \pm standard error of the mean $(\mathrm{SEM}) ; \quad{ }^{* *} P<0.01$. Fig S4: sequences of the primers used for real-time RT-PCR analysis. (Supplementary Materials) 


\section{References}

[1] P. Guo, R. Harding, and I. J. Higginson, "Palliative care needs of heart failure patients in China: putting people first," Current Opinion in Supportive and Palliative Care, vol. 12, no. 1, pp. 10-15, 2018.

[2] J. R. Teerlink, B. A. Davison, G. Cotter et al., "Effects of serelaxin in patients admitted for acute heart failure: a metaanalysis," European Journal of Heart Failure, vol. 22, no. 2, pp. 315-329, 2020.

[3] A. Carbone and A. Gloghini, "Subclassifying peripheral T-cell lymphoma NOS," Blood, vol. 134, no. 24, pp. 2120-2121, 2019.

[4] H. Babaei, N. Razmaraii, G. Assadnassab et al., "Ultrastructural and echocardiographic assessment of chronic doxorubicin-induced cardiotoxicity in rats," Archives of Razi Institute, vol. 75, no. 1, pp. 55-62, 2020.

[5] V. K. Todorova, I. Makhoul, J. Wei, and V. S. Klimberg, "Circulating miRNA profiles of doxorubicin-induced cardiotoxicity in breast cancer patients," Annals of Clinical and Laboratory Science, vol. 47, no. 2, pp. 115-119, 2017.

[6] J. Sui, M. Zhao, Y. Yang et al., “Acid-labile polysaccharide prodrug via lapatinib-sensitizing effect substantially prevented metastasis and postoperative recurrence of triple-negative breast cancer," Nanoscale, vol. 12, no. 25, pp. 13567-13581, 2020.

[7] J. Wen, L. Zhang, J. Wang et al., “Therapeutic effects of higenamine combined with [6]-gingerol on chronic heart failure induced by doxorubicin via ameliorating mitochondrial function," Journal of Cellular Molecular Medicine, vol. 24, no. 7, pp. 4036-4050, 2020.

[8] C. Nabhan, M. Byrtek, A. Rai et al., "Disease characteristics, treatment patterns, prognosis, outcomes and lymphomarelated mortality in elderly follicular lymphoma in the United States," British Journal of Haematology, vol. 170, no. 1, pp. 8595, 2015.

[9] L. Zhao, Y. Qi, L. Xu et al., "MicroRNA-140-5p aggravates doxorubicin-induced cardiotoxicity by promoting myocardial oxidative stress via targeting Nrf2 and Sirt2," Redox Biology, vol. 15, pp. 284-296, 2018.

[10] D. Chihara, J. R. Westin, Y. Oki et al., "Management strategies and outcomes for very elderly patients with diffuse large B-cell lymphoma," Cancer, vol. 122, no. 20, pp. 3145-3151, 2016.

[11] M. M. McGuckin, S. L. Giesy, A. N. Davis et al., "The acute phase protein orosomucoid 1 is upregulated in early lactation but does not trigger appetite-suppressing STAT3 signaling via the leptin receptor," Journal of Dairy Science, vol. 103, no. 5, pp. 4765-4776, 2020.

[12] R. Fandiño-Vaquero, A. Fernández-Trasancos, E. Álvarez et al., "Orosomucoid secretion levels by epicardial adipose tissue as possible indicator of endothelial dysfunction in diabetes mellitus or inflammation in coronary artery disease," Atherosclerosis, vol. 235, no. 2, pp. 281-288, 2014.

[13] F. Ren, Y. Chen, Y. Wang et al., "Comparative serum proteomic analysis of patients with acute-on-chronic liver failure: alpha-1-acid glycoprotein maybe a candidate marker for prognosis of hepatitis B virus infection," Journal of Viral Hepatitis, vol. 17, no. 11, pp. 816-824, 2010.

[14] H. Z. Zhu, W. J. Zhou, Y. F. Wan, K. Ge, J. Lu, and C. K. Jia, "Downregulation of orosomucoid 2 acts as a prognostic factor associated with cancer-promoting pathways in liver cancer," World Journal of Gastroenterology, vol. 26, no. 8, pp. 804$817,2020$.
[15] H. Higuchi, D. Kamimura, J. J. Jiang et al., "Orosomucoid 1 is involved in the development of chronic allograft rejection after kidney transplantation," International Immunology, vol. 32, no. 5, pp. 335-346, 2020.

[16] J. J. Wan, P. Y. Wang, Y. Zhang et al., "Role of acute-phase protein ORM in a mice model of ischemic stroke," Journal of Cellular Physiology, vol. 234, no. 11, pp. 20533-20545, 2019.

[17] X. Fang, H. Wang, D. Han et al., "Ferroptosis as a target for protection against cardiomyopathy," Proceedings of the National Academy of Sciences of the United States of America, vol. 116, no. 7, pp. 2672-2680, 2019.

[18] H. Lin, J. Zhang, T. Ni et al., "Yellow wine polyphenolic compounds prevents doxorubicin-induced cardiotoxicity through activation of the Nrf2 signalling pathway," Journal of Cellular Molecular Medicine, vol. 23, no. 9, pp. 6034-6047, 2019.

[19] S. N. S. Ahmad, D. Sanajou, A. Kalantary-Charvadeh et al., “ $\beta$ LAPachone ameliorates doxorubicin-induced cardiotoxicity via regulating autophagy and $\mathrm{Nrf} 2$ signalling pathways in mice," Basic Clinical Pharmacology Toxicology, vol. 126, no. 4, pp. 364-373, 2020.

[20] M. Chen, V. P. Samuel, Y. Wu et al., "Nrf2/HO-1 mediated protective activity of genistein against doxorubicin-induced cardiac toxicity," Journal of Environmental Pathology, Toxicology and Oncology, vol. 38, no. 2, pp. 143-152, 2019.

[21] L. Rochette, C. Guenancia, A. Gudjoncik et al., "Anthracyclines/trastuzumab: new aspects of cardiotoxicity and molecular mechanisms," Trends in Pharmacological Sciences, vol. 36, no. 6, pp. 326-348, 2015.

[22] D. Liu, Z. Ma, S. di et al., "AMPK/PGC1 $\alpha$ activation by melatonin attenuates acute doxorubicin cardiotoxicity via alleviating mitochondrial oxidative damage and apoptosis," Free Radical Biology \& Medicine, vol. 129, pp. 59-72, 2018.

[23] M. C. Sunitha, R. Dhanyakrishnan, B. PrakashKumar, and K. G. Nevin, "p-Coumaric acid mediated protection of H9c2 cells from doxorubicin-induced cardiotoxicity: involvement of augmented Nrf2 and autophagy," Biomedicine \& Pharmacotherapy, vol. 102, pp. 823-832, 2018.

[24] M. Räsänen, J. Degerman, T. A. Nissinen et al., "VEGF-B gene therapy inhibits doxorubicin-induced cardiotoxicity by endothelial protection," Proceedings of the National Academy of Sciences of the United States of America, vol. 113, no. 46, pp. 13144-13149, 2016.

[25] L. Zhou, R. Li, C. Liu et al., "Foxo3a inhibits mitochondrial fission and protects against doxorubicin-induced cardiotoxicity by suppressing MIEF2," Free Radical Biology \& Medicine, vol. 104, pp. 360-370, 2017.

[26] L. Bharathi Priya, R. Baskaran, C. Y. Huang, and V. Vijaya Padma, "Neferine modulates IGF-1R/Nrf2 signaling in doxorubicin treated H9c2 cardiomyoblasts," Journal of Cellular Biochemistry, vol. 119, no. 2, pp. 1441-1452, 2018.

[27] A. T. Dinkova-Kostova and A. Y. Abramov, "The emerging role of Nrf2 in mitochondrial Function," Free Radical Biology \& Medicine, vol. 88, Part B, pp. 179-188, 2015.

[28] K. M. Holmström, R. V. Kostov, and A. T. Dinkova-Kostova, "The multifaceted role of Nrf2 in mitochondrial function," Current Opinion in Toxicology, vol. 1, pp. 80-91, 2016.

[29] I. G. Ryoo and M. K. Kwak, "Regulatory crosstalk between the oxidative stress-related transcription factor Nfe212/Nrf2 and mitochondria," Toxicology and Applied Pharmacology, vol. 359, pp. 24-33, 2018. 
[30] R. Dhingra, V. Margulets, S. R. Chowdhury et al., "Bnip3 mediates doxorubicin-induced cardiac myocyte necrosis and mortality through changes in mitochondrial signaling," Proceedings of the National Academy of Sciences of the United States of America, vol. 111, no. 51, pp. E5537E5544, 2014.

[31] T. Zhang, Y. Zhang, M. Cui et al., "CaMKII is a RIP3 substrate mediating ischemia and oxidative stress induced myocardial necroptosis," Nature Medicine, vol. 22, no. 2, pp. 175-182, 2016. 